\title{
Nurse Involvement in End of Life Decision Making in the Intensive Care Unit: A Literature Review
}

\author{
Arif Imam Hidayat \\ Master of Nursing Science Student (International \\ Program), Faculty of Nursing, Prince of Songkla \\ University, Thailand \\ hidayat.al.fikri@gmail.com
}

\author{
Waraporn Kongsuwan \\ Associate Professor, Adult and Elderly Nursing \\ Department, Faculty of Nursing, Prince of \\ Songkla University \\ Hat Yai, Thailand \\ waraporn_kongsuwan@yahoo.co.uk
}

\author{
Kittikorn Nilmanat \\ Associate Professor, Adult and Elderly Nursing \\ Department, Faculty of Nursing, Prince of \\ Songkla University \\ Hat Yai, Thailand \\ kittikorn.n@psu.ac.th
}

\begin{abstract}
This article reviews the evidence related to nurse involvement in the end of life (EOL) decision making in the Intensive Care Unit (ICU). This study was conducted by analyzing 53 scholarly papers which met the inclusion criteria. Data were searched through CINAHL, Science Direct, ProQuest, Wiley Online Library, and PubMed. The keywords used were "ICU Nurse", combined with "end of life", "decision making" and "end of life decision making". The databases of PubMed, ProQuest, CINAHL, Wiley Online, Ovid, and Science Direct were used to search for the relevant articles. The results of the analysis present the definition of end of life decision making, type of end of life decision making, persons involved in end of life decision making in the ICU, process of end of life decision making in the ICU, a nurse's role in end of life decision making, and factors influencing end of life decision making in the ICU. The results of this study are important to develop an understanding regarding nurse involvement in end of life decision making in the ICU. Keywords: decision making; end of life; intensive care unit
\end{abstract}

\section{INTRODUCTION}

Healthcare providers who work in the Intensive Care Unit (ICU) focus on delivering care to patients during their acute threats and restoring their quality of lives [50]. Approximately $20 \%$ of patients die while receiving care in an ICU [35] which causes the ICU to have the highest mortality rate compared with other units in the hospital. The majority of patients died after end of life (EOL) decision making [33] which makes EOL decision making become a crucial component of nursing care in the ICU.

DOI: $10.5176 / 2345-7198 \_5.1 .5$

ISSN 2345-7198

CThe Author(s) 2018. This article is published with open access by the GSTF
Nurses in the ICU inevitably become a substantial component during EOL decision making. Nurses are often concerned with doing their best for the patient and the patient's family during EOL decision making and perceive that they have full responsibility regarding the patient's condition which can increase psychological problems among ICU nurses. Profound knowledge regarding nurse involvement in EOL decision making is needed to increase the quality of EOL decision making in the ICU. For an understanding in this area, a literature review was undertaken.

\section{METHODS}

The data of this literature review were collected from searches on databases and manual searching. The databases of PubMed, ProQuest, CINAHL, Wiley Online, Ovid, and Science Direct were used to search for relevant articles using the search terms "ICU Nurse", combined with "end of life", "decision making", and "end of life decision making".

The articles used in this study were limited to articles published in English during the years 20052017. The relevant articles were graded for the level of evidence using the Joanna Briggs Institute for Evidence-Based Nursing and Midwifery (JBI) 2014 levels of evidence. Key information and analysis were extracted into tables which consisted of topics and authors, year of publication, design of the study, 
results, strengths, weaknesses, level of evidence, and implications of the studies.

\section{FINDINGS}

\section{End of Life Decision Making in the ICU}

\section{A. Definition of end of life decision making}

EOL is a condition when the patient is in advanced disability and suffering from a progressive disease which is getting worst during the last 6 to 12 months of the patient's life [16]. During the EOL, the patient often experiences physical symptoms such as shortness of breath, chronic weakness, secondary to pain, and tingling sensation in the extremities due to neurological problems. Psychological impairment which can lead to depression and dementia also occurs often when the patient is dying due to sensory changes [31].

EOL decision making can be considered as a reliable process to analyze all available alternatives of care for the patient to decide on the patient's care during their EOL stage [5]. EOL decision making requires a collaborative process involving not only the healthcare providers in the ICU but the patient and patient's family as well as deciding what kind of treatment will or will not be used during the patient's EOL stage $[48,49]$. Thereby EOL decision making is a collaborative process which involves the healthcare providers, the patient, and the patient's family to consider the direction of care for the patient during the threatening condition.

\section{B. Issues of end of life decision making in the ICU}

- Advance directives

Advance directives are products of advanced care planning which is a dynamic process to identify the patient's preferences during the final stage of life [18, 43]. Advance directives such as living wills and a durable power of attorney for healthcare can be made only when the patient is still capable of making decisions. An advance directive can give the patient a chance to communicate their preferences in writing before some critical condition occurs [39, 49]. Several factors such as a nurse's attitudes on death and religion can influence their involvement while conducting advance directives [28]. Further study is needed to understand the effect of spirituality during nurse involvement in advance directives as a part of EOL decision making [38].

- Withholding or withdrawing therapy

Withholding or withdrawing treatment is a common issue for discussion in the ICU. Withholding treatment can be defined as a decision to not start or continue intervention when the treatment is futile, and withdrawing treatment is a decision to stop treating the patient when the patient's prognosis is poor and the treatment will not benefit the patient $[21,36]$. The withholding or withdrawing of life sustaining therapies can be considered in some circumstances, such as when the benefits for the patient have been exhausted, when life sustaining therapy no longer meets treatment goals or when the topic is rendered by the patient for discussion [31]. Nurses around the world have different perspectives regarding whether they should be involved in EOL decision making, especially regarding withholding or withdrawing treatment [4, 22, 30]. Different perspectives are determined possibly by the level of knowledge and education of the nurses regarding EOL decision making.

- Euthanasia

Euthanasia can be defined as a conscious act to administer medication or other intervention with the intention of causing the patient's death [25, 37]. Euthanasia is considered illegal in almost all countries and unacceptable and is likened to an assisted suicide event even though informed consent is received either from the patient or the patient's family $[37,46]$. Some nurses in the ICU believe that euthanasia is an unethical process and against their religious beliefs [46]. Some healthcare providers in the ICU often have misconceptions regarding euthanasia and have difficulty differentiating between euthanasia and withholding or withdrawing treatment [17, 25]. 
C. Persons involved in end of life decision making in the ICU

The current literature reviewed mentioned that the patient's involvement during EOL decision making in the ICU is very rare due to their threatening condition. A collaborative process during EOL decision making between the patient's family, physician, nurses, and a spiritual advisor is crucial to find the final decision for the patient's care. End of life decision making should involve the patient's family, a healthcare provider, and also allied health workers to ensure that the quality of end of life decision making respects the autonomy of the patient and the patient's family.

- Patient's family

The patient's family is often involved during end of life decision making in the ICU, especially when the patient is unable to express their preferences. A study by Quinn, Schmitt, Baggs, Norton, Dombeck, and Sellers [32] explained that the patient's family can be engaged in several informal roles during end of life decision making in the ICU such as, primary caregiver, primary decision maker, family spokesperson, outof-towner, patient's wish expert, protector, vulnerable member, and healthcare expert.

The order of a patient's family typically starts from the spouse, followed by adult children, and then other family members. They will commonly make the decision regarding the patient's condition in the ICU although this ranking can be different in different countries [10]. The family members who are involved in EOL decision making should be competent persons, which means they should be sufficiently capable to understand the conditions and consider the information and consequences of the decision that they make [37].

- Physician

During this process, the physician should be able to respect the patient and the family's autonomy and provide tailored care to the patient by informing them clearly regarding the situation and empower them to communicate their feelings and perspectives concerning the treatment [53]. Good communication between the physician and the family can prevent violating the patient's quality of life and can increase the family's satisfaction during EOL decision making [53].

- Nurses

Nurses have the role of providing clinical information about the patient's condition to the patient or to the patient's family [8]. Nurses also should be able to facilitate a collaborative process between the patient's family and the healthcare providers during end of life decision making [1]. During EOL decision making, the process is often complex and involves sensitive information which requires the nurse to always respect the patient and the family's privacy and dignity [12].

- Spiritual advisor

A spiritual advisor is very important during end of life decision making to support the patient and the patient's family when the patient is still alive and the support should continue after the death of the patient [1)] Even though nurses try to conduct the role as a spiritual advisor, they often face difficulty due to other work related activities during their working hours [26]. Spiritual advisors can help when a patient wants to consider the issue of EOL decision making by giving consideration related to how religion might view the end of life decision making. The spiritual advisor can also improve the quality of end of life decision making by facilitating patients and their families to achieve the good death in the process by respecting the patient's spiritual and cultural values [47]

\section{Process of end of life decision making in the ICU}

The process of EOL decision making is a cyclic and dynamic process that consists of assessment, disclosure, discussion, and consensus building involving not only a healthcare provider but also the patient and family. This process can last anywhere from hours to weeks or months.

- Assessment

The assessment process regarding the preference of care for the patient should be 
conducted from the time of patient's admission. Unfortunately a patient's condition in the ICU often falls into sudden or unexpected deterioration and makes them unable to participate during EOL decision making. This condition increases the involvement of the family during discussions that involve decision making on behalf of the patient [41]. Assessments should be conducted by the healthcare team which can include the information needs of the patient and family, and the cultural and religious background to address a tailored intervention during EOL decision making. [12].

\section{- Disclosure}

The patient and the patient's family have the right to receive clear and honest information regarding the patient's condition and prognosis. This information should be given by a healthcare provider who is respected as an expert such as a physician [1] based on the assessments of the physician and nurses regarding the patient's condition [2]. Good and honest communication between the healthcare providers, patients, and the patient's family can create trust to defuse tension, and can help the patient's family to synthesize the information and prevent misconception [12, 21].

\section{- Discussion}

Discussions should be conducted when the patient is still competent to decide on the appropriate treatment. If the patient is in an unconscious condition, the family can be invited to make a decision on behalf of the patient. During this process, a meeting between the healthcare provider and the patient's family is crucial to assure that the patient's family has enough understanding regarding the patient's condition. This is important to help the patient's family to reach a consensus, ease burdens surrounding EOL decision making for the family, and reduce distress for the patient's family [10]. The nurse and other healthcare providers should be able to assess the family's information needs and select the important information for the family to help them understand the situation [12]. This intervention is crucial to tailoring information and building rapport with the family [1].

Even though the literature mentions the importance of communication skills during discussions, the nursing literature does not mention the most effective discussion strategies to help the family during EOL decision making [1].

- Documentation

Conformity between the preferences of care of the family or patients and the final care that they receive is crucial to ensure high quality care during EOL decision making [41]. The summarized decision should consist of several important pieces of information such as the medical facts including the prognosis, persons involved in the EOL decision making, patient's wishes if known, goal of the treatments, details of the treatments provided and the treatments to be withdrawn or withheld [1]. The final decision made by the family or the patient regarding EOL treatment during the patient's EOL stage should be documented and delivered to all team members to prevent miscommunication with the family or among the staff personnel, and errors in codes [40].

\section{Nurse Involvement in EOL Decision Making in the ICU}

\section{A. Nurses' roles in end of life decision making}

Based on previous studies, the roles of nurses in end of life decision making in the ICU included educator, advocator, collaborator, and supporter.

\section{- Educator}

The educational process during end of life decision making requires a nurse to be an educator to clarify all information that the patient's family members need to understand the situation. When a patient's family has a good understanding regarding the patient's prognosis, they can be well prepared for the decision making process 
[2]. During the educational process in the end of life decision making, the patient's family can get clear and honest information on the patient's healing possibilities and also possible intervention for the patient [5].

- Advocator

Advocating is one role of nurses during EOL decision making in the ICU. Nurses are expected to be able to speak on behalf of the family to the doctor and on behalf of the patient to the family [1]. Nurses can help the patient's family by understanding the implications of decisions, helping the patient's family to speak up concerning their perceptions and their confusion regarding the patient's condition. When a patient's family receives sufficient information regarding the patient's condition from an early stage, they tend to make decisions more easily during the decision making process [2]. Unfortunately, the failure of nurses to identify the stages of grieving during EOL decision making and lack of knowledge regarding this process often became barriers to be an advocate for the patient and family during EOL decision making $[1,19]$.

\section{- Collaborator}

Nurses should be able to work in a team to fulfill their role as part of the healthcare team. Collaboration between the physician and the nurse is crucial to improve communication between the healthcare professionals and the family during the decision making process [14]. Good collaboration between the healthcare providers is also very important in the decision making process to avoid the emotional burden of providing care and decrease professional distress by receiving support from the colleagues [26].

- Supporter

Adams, Bailey, Anderson, and Docherty [2] explained that nurses have a role in EOL care by building trust in relationships with the family members and by demonstrating empathy for patients, family members, and physicians as they navigate the EOL decision making process. The role of the nurses to provide supportive family care is expected to bring comfort for the patient's family. This can be achieved by meeting the family upon the patient's admission and continuing to meet until the patient reaches a critical condition in order to understand the feelings and expectations of the patient's family [51].

B. Factors influencing the nurses involvement in end of life decision making in the ICU

- Knowledge and experience of nurses

Even though the occurrence of end of life decision making is increasing, nurses often did not have confidence that they were well prepared to help the patients and their families at end of life decision making [49]. Lack of training and experience in nurses in the ICU can hinder the provision of high quality care for patients and their families during end of life decision making [52]. Lack of experience can also cause nurses to have low self-confidence when dealing with end of life decision making [34].

- Patient's condition

During the patient's EOL stage, a patient's family often considers the EOL decision based on the patient's physical appearance, such as facial expression, skin color, and synchrony with the ventilator. The patient's previous condition such as age, athletic activity, and history of illnesses were also considered by the patient's family before taking a decision [7]. Nurses also considered the patient's condition before the initiation of discussions regarding EOL decision making. When a patient's condition remains unstable, the healthcare team tends to delay the EOL decision making, and both the doctor and nurses reported that they felt moral distress when they had different perspectives on the patient's prognosis or the value of suffering [27].

- Belief

A nurse's beliefs are one of the factors that can be a barrier during the end of life decision making process [6]. End of life decision making is still a controversy based on religious points of view and nurses who continually face this condition can 
experience burnout symptoms. Nurses in the ICU often feel that they have full responsibility regarding their patient's condition and this perception tends to make them susceptible to stress and depression [44]. The literature also explains that nurses in the ICU also perceive themselves as a murderer when they have to be involved in EOL decision making [24]. This contrast between the beliefs of the nurses and responsibility could lead to emotional distress which can increase burnout syndrome, low work satisfaction, physical and psychological problems, and could decrease the patient's safety [13, 29, 45]

- Communication

Communication is one of the most common problems in end of life decision making. Ineffective communication can induce problems among nurses, physicians, and a patient's family because the information regarding the patient's condition is not clear and often inconsistent [3]. Communication problems between the nurse and other healthcare team members during EOL decision making also have negative impacts by inducing intra-team conflict which is related to medical errors in patients [15].

- Culture

The EOL decision making process is an emotional issue for the patient, patient's family, and healthcare provider [19]. Culture can have different effects on the responses during EOL decision making and the issues become more complicated when religion is considered [42]. The nurses must understand the distinction and influence of culture on the behavior of the patient and the patient's family by doing a comprehensive assessment and respecting each subject as an individual with his or her own uniqueness $[9,11]$.

\section{CONCLUSION}

EOL decision making is a process which is conducted to consider the patient's treatment during their end of life stage. Nurses in the ICU, together with other healthcare providers, should be able to deliver care during end of life decision making and carry out their role for the patient and the patient's family as well. Nurses have very important roles during end of life decision making such as educator, advocator, collaborator, and supporter. Nurses can facilitate the patient's families during end of life decision making to get clear information and prevent miscommunication. Some factors that can influence a nurse's involvement in EOL decision making are knowledge and experience of the healthcare providers, the patient's condition, beliefs, communication, and culture.

From the literature review it can be summarized that nurses in the ICU have a crucial role as a member of a collaborative team during EOL decision making. Unfortunately, nurses often have a low level of self-confidence during their involvement in EOL decision making due to their lack of knowledge and experience. Nurses often have to deal with dilemmatic conditions when they have contradictions between their beliefs and their role during EOL decision making. A nurse's understandings of the cultural and religion values of the patient and patient's family are also important to increase the quality of care during EOL decision making in the ICU. However, the perception of nurses regarding their beliefs in EOL decision making is not clearly understood. The findings of this literature review will be useful in providing knowledge on the involvement of nurses in end of life decision making in the ICU and can be used as knowledge to improve the quality of care for the patients and families of patients in the end of life decision making context in the ICU.

\section{ACKNOWLEDGMENT}

The authors express gratitude to the Prince of Songkla University for the financial support provided of this study through Thailand's Education Hub for Southern Region of ASEAN Countries (TEH-AC) scholarship.

\section{REFERENCES}

[1]. Adams, J. A. (2013). Family perspectives of nursing strategies to facilitate transition from curative to palliative care in the intensive care unit (Doctoral dissertation, Duke University). Retrieved from

https://dukespace.lib.duke.edu/dspace/handle/10161/8055 
[2]. Adams, J. A., Bailey, D. E., Anderson, R. A., \& Docherty, S. L. (2011). Nursing roles and strategies in end-of-life decision making in acute care: A systematic review of the literature. Nursing Research and Practice. 2011, 1-15. doi: $10.1155 / 2011 / 527834$

[3]. Aslakson, R. A., Wyskiel, R., Thornton, I., Copley, C., Shaffer, D., Zyra, M. ... \& Pronovost, P. J. (2012). Nurse-perceived barriers to effective communication regarding prognosis and optimal end-of-life care for surgical ICU patients: A qualitative exploration. Journal of Palliative Medicine, 15, 910-915. doi: 10.1089/jpm.2011.0481

[4]. Badır, A., Topçu, I., Türkmen, E., Göktepe, N., Miral, M., Ersoy, N., \& Akın, E. (2015). Turkish critical care nurses views on end-of-life decision making and practices. Nursing in Critical Care, 21, 334-342. doi:10.1111/nicc.12157

[5]. Baliza, M. F., Bousso, R. S., Poles, K., Santos, M. R. D., Silva, L., \& Paganini, M. C. (2015). Factors influencing intensive care units nurses in end-of-life decisions. Revista da Escola de Enfermagem da USP (Magazine of the School of Nursing of USP), 49, 572-579. doi: 10.1590/S0080-623420150000400006

[6]. Borhani, F., Hosseini, S. H., \&Abbaszadeh, A. (2014). Commitment to care: A qualitative study of intensive care nurses' perspectives of end - of - life care in an Islamic context. International Nursing Review, 61(1), 140-147. doi: 10.1111/inr.12079

[7]. Boyd, E. A., Lo, B., Evans, L. R., Malvar, G., Apatira, L., Luce, J. M., \& White, D. B. (2010). "It's not just what the doctor tells me:" Factors that influence surrogate decision-makers' perceptions of prognosis. Critical Care Medicine, 38, 12701275. doi: 10.1097/CCM.0b013e3181d8a217

[8]. Brooks, L. A., Manias, E., \& Nicholson, P. (2017). Barriers, enablers and challenges to initiating end-of-life care in an Australian intensive care unit context. Australian Critical Care, 30(3), 161-166. doi:10.1016/j.aucc.2016.08.001

[9]. Bullock, K. (2011). The influence of culture on end-of-life decision making. Journal of Social Work in End-of-Life \& Palliative Care, 7(1), 83-98. doi: $10.1080 / 15524256.2011 .548048$

[10]. Cai, X., Robinson, J., Muehlschlegel, S., White, D. B., Holloway, R. G., Sheth, K. N., \& Hwang, D. Y. (2015). Patient preferences and surrogate decision making in neuroscience intensive care units. Neurocritical Care, 23(1), 131-141. doi:10.1007/s12028-015-0149-2.

[11]. Coolen, P. R. (2012). Cultural relevance in end-of-life care. Retrieved from https://ethnomed.org/clinical/end-oflife/cultural-relevance-in-end-of-life-care

[12]. Coombs M.A., Parker R., Ranse K., Endacott R. \& Bloomer M.J. (2016) An integrative review of how families are prepared for, and supported during withdrawal of life-sustaining treatment in intensive care. Journal of Advanced Nursing, 73(1), 39-55. doi: 10.1111/jan.13097

[13]. Donovan, R. O., Doody, O., \& Lyons, R. (2013). The effect of stress on health and its implications for nursing. British Journal of Nursing, 22, 969-973. doi:10.12968/bjon.2013.22.16.969

[14]. Efstathiou, N., \& Clifford, C. (2011). The critical care nurse's role in end - of - life care: issues and challenges. Nursing in Critical Care, 16(3), 116-123. doi: 10.1111/j.14785153.2010.00438.x
[15]. Fassier, T., \& Azoulay, E. (2010).Conflicts and communication gaps in the intensive care unit. Current Opinion in Critical Care, 16, 654-665. doi: 10.1097/MCC.0b013e32834044fo

[16]. General Medical Council. (2010). Treatment and care towards the end of life: good practice in decision making. General Medical Council. Retrieved from http://www.gmcuk.org/static/documents/content/Treatment_and_care_towards _the_end_of_life_-_English_1015.pdf

[17]. Goligher, E. C., Ely, E. W., Sulmasy, D. P., Bakker, J., Raphael, J., Volandes, A. E., Downar, J. (2017). Physicianassisted suicide and euthanasia in the ICU. Critical Care Medicine, 45(2), 149-155. doi:10.1097/ccm.0000000000001818

[18]. Gutierrez, K. M. (2012). Advance directives in an intensive care unit. Critical Care Nursing Quarterly, 35, 396-409. doi:10.1097/cnq.0b013e318268fe35

[19]. Hebert, K., Moore, H., \& Rooney, J. (2011). The nurse advocate in end-of-life care. The Ochsner Journal, 11, 325 329. Retrieved from https://www.ncbi.nlm.nih.gov/pmc/articles/PMC3241064/pdf/i 1524-5012-11-4-325.pdf

[20]. Hildén, H. M., \& Honkasalo, M. L. (2006). Finnish nurses' interpretations of patient autonomy in the context of end-of-life decision making. Nursing Ethics, 13(1), 41-51. doi: https://doi.org/10.1191/0969733006ne856oa

[21]. Hoel, H., Skjaker, S. A., Haagensen, R., \& Stavem, K. (2014) Decisions to withhold or withdraw life-sustaining treatment in a Norwegian intensive care unit. Acta Anaesthesiologica Scandinavica, 58, 329-336. doi:10.1111/aas.12246

[22]. Inghelbrecht, E., Bilsen, J., Mortier, F., \& Deliens, L. (2009). Nurses' attitudes towards end-of-life decisions in medical practice: A nationwide study in Flanders, Belgium. Palliative Medicine, 23, 649-658. doi: https://doi.org/10.1177/0269216309106810

[23]. Jordan, P. J., Clifford, I., \& Williams, M. (2014). The experiences of critical care nurses with regard to end-of-life issues in the intensive care unit. Africa Journal of Nursing and Midwifery, 16(2), 71-84. http://hdl.handle.net/10520/EJC169758

[24]. Kisorio, L. C., \& Langley, G. C. (2016). Intensive care nurses' experiences of end-of-life care. Intensive and Critical Care Nursing, 33, 30-38. doi:10.1016/j.iccn.2015.11.002

[25]. Kranidiotis, G., Ropa, J., Mprianas, J., Kyprianou, T., \& Nanas, S. (2015). Attitudes towards euthanasia among Greek intensive care unit physicians and nurses. Heart \& Lung: The Journal of Acute and Critical Care, 44, 260-263. doi:10.1016/j.hrtlng.2015.03.001

[26]. Kryworuchko, J., Hill, E., Murray, M. A., Stacey, D., \& Fergusson, D. A. (2013). Interventions for shared decision making about life support in the intensive care unit: A systematic review. Worldviews on Evidence - Based Nursing, 10(1), 3-16. doi: 10.1111/j.1741-6787.2012.00247.x

[27]. Kryworuchko, J., Strachan, P. H., Nouvet, E., Downar, J., \& You, J. J. (2016). Factors influencing communication and decision-making about life-sustaining technology during serious illness: a qualitative study. BMJ (British Medical Journal) Open, 6(5). 1-11 doi:10.1136/bmjopen-2015-010451

[28]. Lynn, T., Curtis, A., \& Lagerwey, M. D. (2015). Association between attitude toward death and completion of advance 
directives. OMEGA - Journal of Death and Dying, 74(2), 1-19. doi:10.1177/0030222815598418

[29]. Martins, J. T., \& Maria Lúcia Do Carmo Cruz Robazzi. (2009). Nurses work in intensive care units: Feelings of suffering. Revista Latino-Americana de Enfermagem (Latin American Journal of Nursing), 17(1), 52-58. doi:10.1590/s0104-11692009000100009

[30]. MengJie, L., HouXiu, Z., ChangBi, L., FuYu, H., SiLin, Z., \& JingCi, Z. (2015). End-of-life decision-making experiences and influencing factors reported by intensive care unit medical and nursing staff members in southwestern china: A Qualitative Study. Journal of Hospice \& Palliative Nursing, 17, 544-550. doi: 10.1097/NJH.0000000000000196

[31]. Papadimos, T., Tripathi, R., Rosenberg, A., Maldonado, Y., \& Kothari, D. (2011). An overview of end-of-life issues in the intensive care unit. International Journal of Critical Illness and Injury Science, 1(2), 138-146. doi:10.4103/22295151.84801

[32]. Quinn, J. R., Schmitt, M., Baggs, J. G., Norton, S. A., Dombeck, M. T., \& Sellers, C. R. (2012). Family members' informal roles in end-of-life decision making in adult intensive care units. American Journal of Critical Care, 21(1), 43-51. doi: $10.4037 /$ ajcc 2012520

[33]. Ranse, K., Yates, P., \& Coyer, F. (2012). End-of-life care in the intensive care setting: a descriptive exploratory qualitative study of nurses' beliefs and practices. Australian Critical Care, 25(1), 4-12. doi: 10.1016/j.aucc.2011.04.004

[34]. Raphael, D., Waterworth, S., \& Gott, M. (2014). The role of practice nurses in providing palliative and end-of-life care to older patients with long-term conditions. International Journal of Palliative Nursing, 20,373-379. doi: 10.12968/ijpn.2014.20.8.373

[35]. Romain, M., \& Sprung, C. L. (2014). End-of-life practices in the intensive care unit: the importance of geography, religion, religious affiliation, and culture. Rambam Maimonides Medical Journal, 5(1), 1-7. doi: 10.5041/RMMJ.10137

[36]. Rydvall, A., \& Lynöe, N. (2008). Withholding and withdrawing life-sustaining treatment: a comparative study of the ethical reasoning of physicians and the general public. Critical Care, 12(1), 1-7. doi:10.1186/cc6786

[37]. Schüklenk, U., Delden, J. J., Downie, J., Mclean, S. A., Upshur, R., \& Weinstock, D. (2011). End-of-life decisionmaking in Canada: The report by the royal society of Canada expert panel on end-of-life decision-making. Bioethics, 25, 173. doi:10.1111/j.1467-8519.2011.01939x

[38]. Seale, C. (2010). The role of doctors' religious faith and ethnicity in taking ethically controversial decisions during endof-life care. Journal of Medical Ethics, 36, 677-682. doi:10.1136/jme.2010.036194

[39]. Shapiro, S. P. (2015). Do advance directives direct?. Journal of Health Politics, Policy and Law, 40, 487-530. doi:10.1215/03616878-2888424

[40]. Shekelle, P. G., Wachter, R. M., Pronovost, P. J., Schoelles, K., McDonald, K. M., Dy, S. M., Winters, B. D. (2013). Making health care safer II: an updated critical analysis of the evidence for patient safety practices. Evidence Report/Technology Assessment, (211), 1-2. Retrieved from https://www.ncbi.nlm.nih.gov/pmc/articles/PMC4781147/

[41]. Sinuff, T., Dodek, P., You, J. J., Barwich, D., Tayler, C., Downar, J., Heyland, D. K. (2015). Improving end-of-life communication and decision making: The development of a conceptual framework and quality indicators. Journal of Pain and Symptom Management, 49, 1070-1080.

doi:10.1016/j.jpainsymman.2014.12.007

[42]. Steinberg, S. M. (2011). Cultural and religious aspects of palliative care. International Journal of Critical Illness and Injury Science, 1(2), 154-156. doi: 10.4103/22295151.84804

[43]. Stuart, B., Volandes, A., \& Moulton, B. W. (2017). Advance care planning: Ensuring patients' preferences govern the care they receive. Generations, 41(1), 31-36. Retrieved from http://www.ingentaconnect.com/contentone/asag/gen/2017/000 00041/00000001/art00006\#expand/collapse

[44]. Teixeira, C., Ribeiro, O., Fonseca, A. M., \& Carvalho, A. S. (2013). Ethical decision making in intensive care units: a burnout risk factor? Results from a multicenter study conducted with physicians and nurses. Journal of Medical Ethics. 40(2), 97-103 doi: 10.1136/medethics-2012-100619

[45]. Teng, C., Chang, S., \& Hsu, K. (2009). Emotional stability of nurses: Impact on patient safety. Journal of Advanced Nursing, 65, 2088-2096. doi:10.1111/j.1365-2648.2009.05072.x

[46]. Tepehan, S., Ozkara, E., \& Yavuz, M. F. (2009). Attitudes to euthanasia in ICUs and other hospital departments. Nursing Ethics, 16, 319-327. doi:10.1177/0969733009102693

[47]. The American College of Obstetricians and Gynecologist (ACOG) Committee (2008) Opinion No. 403: End-of-life decision making. Obstetrics \& Gynecology, 111, 1021-1027. doi:10.1097/aog.0b013e3181713d92

[48]. The Royal Australian College of Physicians. (2016). Improving care at the end of life: Our role and responsibilities. Retrieved from https://www.racp.edu.au/docs/default-source/advocacylibrary/pa-pos-end-of-life-position-statement.pdf

[49]. Thelen, M. (2005). End-of-life decision making in intensive care. Critical Care Nurse, 25(6), 28-37. Retrieved from http://ccn.aacnjournals.org/content/25/6/28.full.pdf+html

[50]. Truog, R. D., Campbell, M. L., Curtis, J. R., Haas, C. E., Luce, J. M., Rubenfeld, G. D., \& Kaufman, D. C. (2008). Recommendations for end-of-life care in the intensive care unit: A consensus statement by the American college of critical care medicine. Critical Care Medicine, 36, 953-963. doi: 10.1097/CCM.0B013E3181659096

[51]. Tsaloukidis, N. (2010). Treating ICU patients of final stage and dealing with their family. Health Science Journal, 4(3), 136141. Retrieved from http://www.hsj.gr/medicine/treating953cu-patients-of-final-stage-and-dealing-with-theirfamily.pdf

[52]. Velarde-Garcia, J., Pulido-Mendoza, R., Nieves Moro-Tejedor, M., Miguel Cachon-Perez, J., \& Palacios-Cena, D. (2016). Nursing and end-of-life care in the intensive care unit. Journal of Hospice \& Palliative Nursing, 18(2), 115-123. doi: 10.1097/NJH.000000000000021

[53]. Visser, M., Deliens, L., \& Houttekier, D. (2014). Physicianrelated barriers to communication and patient- and familycentred decision-making towards the end of life in intensive care: A systematic review. Critical Care, 18(6), 1-19 doi:10.1186/s13054-014-0604-z 